\begin{tabular}{|l|l|}
\hline POS1430 & EPIDEMIOLOGY OF LUPUS NEPHRITIS IN \\
BRAZIL: FINDINGS FROM THE MACUNAÍMA \\
STUDY - A NATIONWIDE MULTICENTRIC \\
STUDY
\end{tabular}

M. Abreu ${ }^{1,2}$, O. Monticielo ${ }^{3}$, V. Fernandes ${ }^{4}$, A. Cristovão Maiorano ${ }^{2}$ F. Dos Santos Beserra ${ }^{2}$, F. Lamarao ${ }^{5}$, N. David ${ }^{5}$, B. De Veras ${ }^{5}$, B. Bica ${ }^{1}$, D. Sávio Nunes de Lima ${ }^{6}$, M. Maria Das Chagas Medeiros ${ }^{7} .{ }^{1}$ Universidade Federal do Rio de Janeiro, Faculdade de Medicina, Rio de Janeiro, Brazil ${ }^{2}$ Universidade Federal do Rio de Janeiro, Laboratório Para Pesquisa em Desfechos Centrados no Paciente, Rio de Janeiro, Brazil: ${ }^{3}$ Universidade Federal do Rio Grande do Sul, Serviço de Reumatologia do Hospital de Clínicas de Porto Alegre, Porto Alegre, Brazil; ${ }^{4}$ Universidade de Cuiabá, Department of Medicine, Cuiabá, Brazil; ${ }^{5}$ GlaxoSmithKline, Medical Department, Rio de Janeiro, Brazil; ${ }^{6}$ Universidade Federal do Amazonas, Serviço de Reumatologia do Hospital Universitário Getúlio Vargas, Manaus, Brazil; ' Universidade Federal do Ceará, Faculdade de Medicina, Fortaleza, Brazil

Background: Lupus nephritis (LN) is one of the most serious organic manifestations of systemic lupus erythematosus (SLE). Ethnicity can contribute to disparities in the prevalence and disease activity of $L N$.

Objectives: To assess the prevalence of $L N$ in Brazilian patients with SLE and to determine factors associated with LN activity across the country.

Methods: This cross-sectional study (GSK Study 207353) was carried out through face-to-face interviews and review of medical records (12-month study period). Adult patients with SLE (American College of Rheumatology [ACR] criteria, 1997) were included. Five SLE reference teaching centres were selected: North (NO), Northeast (NE), Midwest (CO), Southeast (SE), and South (SU). Patients with another disease whose morbidity surpassed SLE were excluded. LN was defined as reported in the medical record or history of confirmed renal biopsy; disease activity by pre-defined changes in SLE Disease Activity Index (SLEDAI) or the patient's kidney disease during the study. Activity was assessed during (T0), 6 months before (T6), and 12 months before (T12) the interview. Systemic Lupus International Collaborating Clinics/ACR Damage Index score mapped damage accrual. Two pairings were performed, aiming to discriminate factors associated with LN and its activity, respectively. Matching technique was used to select similar individuals based on propensity scores, obtained from a logistic regression model. A bootstrapping method explored characteristic variables associated with the risk of progressing to LN.

Results: Overall, 300 Brazilian patients with SLE were included in the study. Two groups were paired: $L N$ group $(N=150)$ and non- $L N$ group $(N=141)$. The prevalence of $\mathrm{LN}$ in the paired sample $(\mathrm{N}=291)$ was $51.5 \%$, with a disparity between centres $(p<0.001$; Figure $1 A)$. Most patients were female (LN: $92.7 \%$; non-LN: $94.3 \%$ ) and the mean (standard deviation [SD]) age for the LN and non-LN groups was 39.46 (11.86) and 43.96 (12.18), respectively. History of serositis was associated with the presence of LN (42 [28.0\%] vs 21 [14.9\%] non-LN; $p=0.010$ ). Type IV histological class predominated in both groups, with no disparity between centres. Social disparities were noted between groups. Non-active workers prevailed among the LN group (115 [76.7\%] vs 98 [69.5\%] for non-LN, $p=0.024$ ).

When pairing for disease activity at $\mathrm{T} 12,73(50.3 \%)$ patients with $\mathrm{LN}(\mathrm{N}=145)$ had active disease. There was regional disparity in terms of disease activity (Figure $1 \mathrm{~B})$, with a predominance of active $\mathrm{LN}$ in the NO $(28[68.3 \%])$ and SU (16 [55.2\%], $p=0.026)$. Type IV histological class was the component most associated with active LN (active: 32 [43.8\%]; non-active: 11 [15.3\%], $\mathrm{p}<0.001$ ). Variation in SLEDAI during the study period discriminated between active and non-active LN. The mean (SD) SLEDAI score at T12 was substantially higher in those with active LN compared with non-active LN (7.18 [4.83] vs 2.47 [4.63], $p<0.001)$. As for the pattern of care, corticosteroids users prevailed in those with active LN (62 [84.9\%] vs $45[62.5 \%]$ for non-active LN, $\mathrm{p}=0.004)$. There was no disparity in the use of immunosuppressants, with the exception of cyclophosphamide use, noted among $16(21.9 \%)$ patients with active $L N$ and $6(8.3 \%)$ patients with non-active $L N(p=0.041)$. Psychotropic or anticonvulsant use was higher in patients with non-active LN (32 [44.4\%] vs 17 [23.3\%] patients with active $\mathrm{LN}, \mathrm{p}=0.012$ ). Consultation with a neurologist was verified in $15(20.8 \%)$ patients with non-active $L N$ and $6(8.2 \%)$ with active LN $(p=0.055)$. Hospitalisation occurred in 17 patients with non-active $(23.6 \%)$ and active $(23.3 \%) \mathrm{LN}$.

Conclusion: Disparities in the prevalence of LN and its activity were evident between the regions across Brazil, highlighting differences in clinical factors, regional factors, and patterns of care.

Funding: GSK
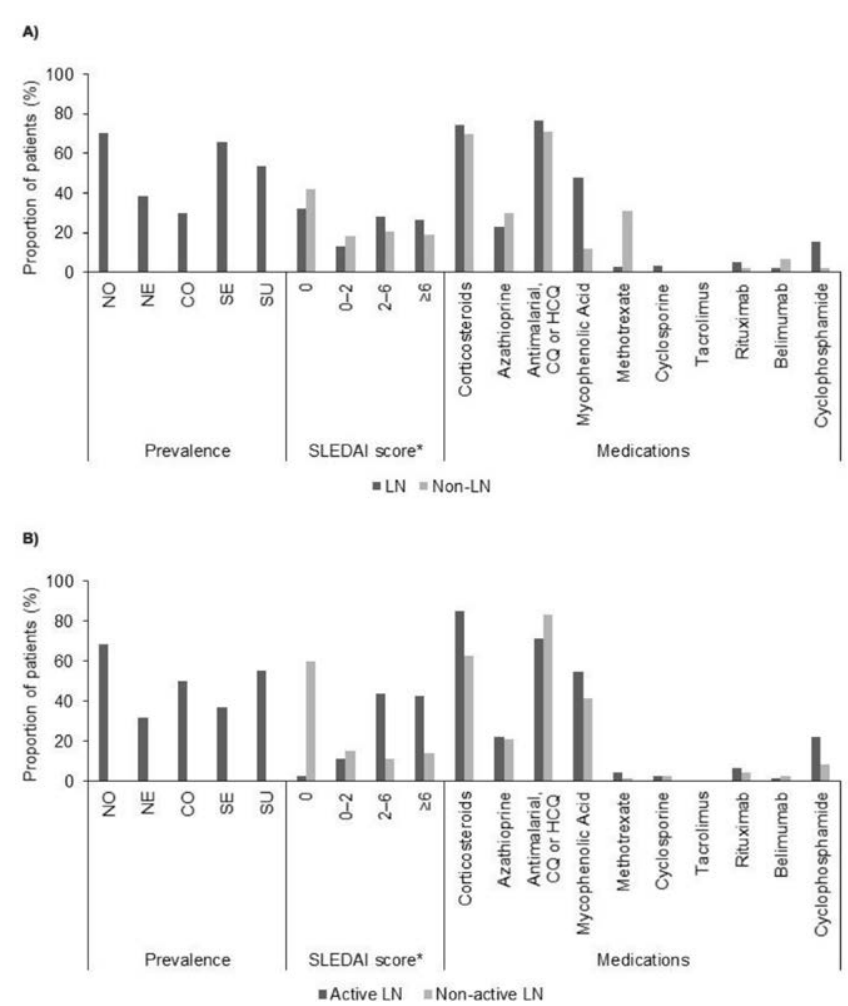

Figure 1. Prevalence of A) $L N$ among regional centres, comparing them to disease activity profile and prescriptive practice, and B) Active and non-active LN according to prescriptive practice $C Q$, chloroquine; $H C Q$, hydroxychloroquine*At $\mathrm{T} 12$

Acknowledgements: Medical writing assistance was provided by Helen Taylor, Fishawack Indicia Ltd., UK, part of Fishawack Health, and was funded by GSK. Disclosure of Interests: Mirhelen Abreu Grant/research support from: GSK, Amgen, Biogen, Libbs, Odirlei Monticielo Speakers bureau: GSK, AbbVie, UCB, Roche, Novartis, Consultant of: GSK, AbbVie, Janssen Vander Fernandes Speakers bureau: Janssen, Novartis, Roche, AbbVie, Pfizer, Grant/research support from: Novartis, GSK, Pfizer, Alexandre Cristovão Maiorano: None declared, Fernando dos Santos Beserra: None declared, Flavia Lamarao Employee of: GSK, Nathalie David Shareholder of: GSK, Employee of: GSK, Bruna de Veras Employee of: GSK, Blanca Bica: None declared, Domingos Sávio Nunes de Lima Grant/ research support from: GSK, Marta Maria das Chagas Medeiros: None declared

DOI: 10.1136/annrheumdis-2021-eular.2336

\section{POS1431 \\ THE ASSOCIATION OF CLINICAL AND STRUCTURAL KNEE OSTEOARTHRITIS WITH PHYSICAL ACTIVITY IN THE MIDDLE-AGED POPULATION: THE NEO STUDY}

S. Terpstra ${ }^{1}$, J. Van der Velde ${ }^{2}$, R. De Mutsert ${ }^{2}$, D. Schiphof ${ }^{3}$, M. Reijnierse $^{4}$ F. Rosendaal5, M. Kloppenburg5,6, M. Loef6. ' ${ }^{1}$ Leiden University Medical Center, Rheumatology, Leiden, Netherlands; ${ }^{2}$ Leiden University Medical Center, Clinical Epidemiology, Leiden, Netherlands; ${ }^{3}$ Erasmus Medical Center, General Practice, Rotterdam, Netherlands; ${ }^{4}$ Leiden University Medical Center, Radiology, Leiden, Netherlands; ${ }^{2}$ Leiden University Medical Center, Clinical Epidemiology, Leiden, Netherlands; ${ }^{1}$ Leiden University Medical Center, Rheumatology, Leiden, Netherlands

Background: Lack of physical activity in individuals with knee OA has shown to be associated with increased cardiovascular risk and mortality. Consequently, physical activity is a potential target for interventions in knee OA. However, most of the available studies concerning physical activity in individuals with knee OA were performed in relatively old populations with an inactive lifestyle. It is unclear 
how previous results can be generalized to other populations with different lifestyle and physical activity habits.

Objectives: To investigate if knee OA is associated with lower physical activity in a general middle-aged Dutch population. Furthermore, to investigate the association of physical activity with patient reported outcomes such as knee pain and function, and health-related quality of life in individuals with knee OA.

Methods: We used cross-sectional data from the Netherlands Epidemiology of Obesity (NEO) study, in which participants aged 45-65 years were included. Clinical knee OA was defined using the ACR criteria. Structural knee OA was defined on MRI using the modified criteria by Hunter et al. in a random subset of 1,285 individuals of our study population.

We assessed knee pain and function with the Knee injury and Osteoarthritis Score (KOOS), and health-related quality of life (HRQoL) with the Short Form (SF)-36. Physical activity (in Metabolic Equivalent of Task (MET) hours per week) was assessed using the Short Questionnaire to Assess Health-enhancing physical activity (SQUASH).

We used linear regression analyses to investigate 1) the association of knee OA with physical activity, and 2) of physical activity with knee pain, function, and HRQoL in participants with clinical knee OA. All analyses were adjusted for age, sex, body mass index (BMI), ethnicity, educational level and comorbidities. To account for possible information bias, we performed a sensitivity analysis to assess the association between clinical knee OA and physical activity measured by an accelerometer in a random subset of $15 \%$ of the study population.

Results: Of 6,212 participants, we observed clinical knee OA in $14 \%$, and structural knee $O A$ in $12 \%$. The general population characteristics and median physical activity of our study population are presented in Table 1. In comparison to participants without knee OA, participants with clinical knee OA had on average $9.60(95 \% \mathrm{Cl} 3.70 ; 15.50)$ MET hours per week more total physical activity (Figure 1). Structural knee OA was associated with 3.97 (-7.82; 15.76) MET hours per week more physical activity, compared with no structural knee OA.

Sensitivity analysis showed a weak positive association of clinical knee OA with physical activity measured by an accelerometer: $2.37(-6.05 ; 10.80)$ MET hours per week more physical activity in participants with clinical knee OA, compared with participants without clinical knee OA.

In the subpopulation of participants with clinical knee OA, physical activity was not associated with knee pain, function or HRQoL.

Conclusion: Knee OA was not associated with lower physical activity in this middle-aged Dutch population. This contrasts previous findings and warrants caution when generalizing physical activity outcomes to other populations. Furthermore, it stresses the need of more insight in the barriers and facilitators of physical activity in the middle-aged population.

Table 1. Characteristics of the NEO study population

\begin{tabular}{lccc}
\hline & & $\begin{array}{c}\text { No clinical knee } \\
\text { OA }\end{array}$ & $\begin{array}{c}\text { Clinical knee } \\
\text { OA }\end{array}$ \\
& $\mathbf{n}=\mathbf{6 , 2 1 4}$ & $\mathbf{8 6 \%}$ & $\mathbf{1 4 \%}$ \\
\hline $\begin{array}{l}\text { General population characteristics } \\
\text { Age (year) }\end{array}$ & $55.7(6.0)$ & $55.4(6.1)$ & $57.5(5.0)$ \\
$\begin{array}{l}\text { Sex (\% women) } \\
\left.\text { BMI (kg/m }{ }^{2}\right)\end{array}$ & 55 & 54 & 67 \\
Comorbidities (\% present) & $26.3(4.4)$ & $26.1(4.3)$ & $27.6(5.1)$ \\
$\begin{array}{l}\text { Physical activity } \\
\text { Total^ (MET-hours per week) }\end{array}$ & 24 & 23 & 32 \\
& 118.8 & 118.4 & 123.5 \\
& $(76.8 ; 155.0)$ & $(76.6 ; 154.4)$ & $(77.8 ; 157.2)$ \\
\hline
\end{tabular}

Numbers represent mean (SD) or percentages. ^median (25th, 75th percentiles). Abbreviations: $\mathrm{OA}=$ osteoarthritis. $\mathrm{BMI}=$ Body Mass Index. $\mathrm{MET}=$ Metabolic Equivalent of Task.

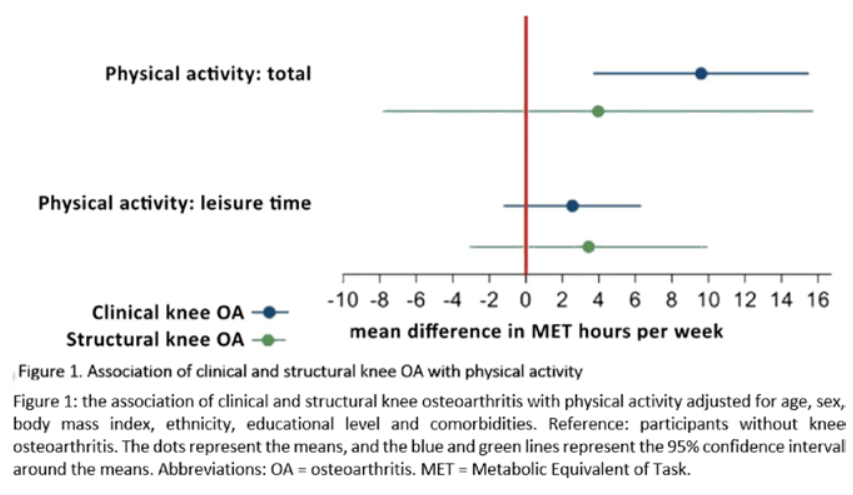

Disclosure of Interests: None declared

DOI: 10.1136/annrheumdis-2021-eular.2363

POS1432
SHORT-TERM RISK OF OSTEOPOROSIS
IN ADULTS TREATED WITH CORTICOSTEROIDS:
AN OBSERVATIONAL STUDY

E. Berni ${ }^{1}$, P. Ambery ${ }^{2}$, S. Adamsson Eryd ${ }^{3}$, S. Jenkins-Jones ${ }^{1}, M$. Brown ${ }^{4}$, C. Astbury ${ }^{5}$, P. Hunt ${ }^{6}$, C. Currie ${ }^{1,7} .{ }^{1}$ Pharmatelligence, Epidemiology, Cardiff, United Kingdom; ${ }^{2}$ AstraZeneca, Late-Stage Development, Cardiovascular, Renal and Metabolism, BioPharmaceuticals $R \& D$, Gothenburg, Sweden; ${ }^{3}$ AstraZeneca, BioPharmaceuticals Medical, CVRM Evidence, Gothenburg, Sweden; ${ }^{4}$ AstraZeneca, Research and Early Development, Respiratory \& Immunology, BioPharmaceuticals R\&D, Boston, United States of America:

${ }^{5}$ AstraZeneca, Research and Early Development, Respiratory \& Immunology, BioPharmaceuticals R\&D, Cambridge, United Kingdom;

${ }^{6}$ AstraZeneca, BioPharmaceuticals Medical, CVRM Evidence, Gaithersburg, United States of America; ${ }^{7}$ Cardiff University, School of Medicine, Cardiff, United Kingdom

Background: Exposure to corticosteroids is recognised to increase the risk of osteoporosis.

Objectives: Our aim was to evaluate patients' short-term absolute risk of osteoporosis over the course of their first continuous exposure to corticosteroids.

Methods: This was an observational study using UK data from the Clinical Practice Research Datalink. Adult patients were selected if exposed to systemic corticosteroids for any condition and had no prior osteoporosis. Non-exposed adults matched on age, sex, and disease burden were selected from the general population. Patients were followed from their first exposure to corticosteroid to the earlier of 90 days following the end of continuous prescribing or for a maximum of three years. Cohorts were categorised by age (18-42 years, 43-67 and 68-92 years), BMI (underweight, normal overweight, obese and obese+) and gender. Absolute risk rates were calculated for each of these categories.

Results: In total, 573,056 exposed patients were matched 1:1 to non-exposed controls. Mean age was 52 years; $57 \%$ were female. The mean and median days' supply were 50.9 and 13 days, respectively. Underweight females aged 68-92 years exposed to corticosteroids had the highest absolute risk of osteoporosis (70.9 per 1000 patient years (PKPY)); in matched non-exposed controls this was 26.6 PKPY. Generally, following their first continuous exposure to corticosteroids, patients taking steroids had greater risk of osteoporosis compared with those in the same age, sex and BMI category never exposed to corticosteroids.

Conclusion: Whilst it is understood that exposure to corticosteroids increases the risk of osteoporosis, there are large differences in risk in accordance with age, sex and BMI. Alternatives to corticosteroids are urgently needed. 